\title{
Amino acetaldehyde conformers: structure and spectroscopic properties
}

\author{
Pilar Redondo ${ }^{\circledR}{ }^{\star}$ Miguel Sanz-Novo, Antonio Largo and Carmen Barrientos \\ Computational Chemistry Group, Departamento de Química Física y Química Inorgánica, Facultad de Ciencias, Universidad de Valladolid, E-47011 \\ Valladolid, Spain
}

Accepted 2019 December 13. Received 2019 December 11; in original form 2019 November 11

\begin{abstract}
We present a computational study of the different conformers of amino acetaldehyde. This molecule is a precursor of glycine and also an isomer of the detected molecules acetaldehyde and methylformamide. In addition, a previous theoretical result shows that amino acetaldehyde could be formed from the gas phase reaction of formamide with $\mathrm{CH}_{5}^{+}$. Different computational approaches, going from density functional theory (DFT) to coupled cluster (CC) calculations, are employed for the characterization of the amino acetaldehyde conformers. We locate four low-lying conformation on the singlet potential energy surface (PES), two with a synperiplanar arrangement of the carboxylic oxygen atom and the $\mathrm{NH}_{2}$ group, and the other two conformers with an anticlinal disposition. All levels of theory predict the conformer with a synperiplanar arrangement and the $\mathrm{H}$ atoms of the $\mathrm{NH}_{2}$ group pointing in the direction of the oxygen, denoted as in-sp-amino acetaldehyde, as the most stable. The viability of the interconversion processes between the four conformers in space is analysed. Relevant spectroscopic parameters to rotational spectroscopy with 'spectroscopic' accuracy at the composite level are reported. Vibrational frequencies and infrared intensities are also computed at the $\mathrm{CC}$ with single and double excitations (CCSD) level including anharmonic corrections. This information could help in the experimental characterization of amino acetaldehyde that could be considered as a good candidate molecule to be searched for in space.
\end{abstract}

Key words: astrobiology-astrochemistry -ISM: general-ISM: molecules.

\section{INTRODUCTION}

Complex organic molecules (COMs), organic species containing carbon, nitrogen, and oxygen atoms and having six of more atoms, are considered as possible precursors of prebiotic molecules in the interstellar medium (ISM). These molecules under prebiotic conditions could play a crucial role in the synthesis of biomolecules, the building blocks of life (Quan et al. 2016). The increase on the number and complexity of the detected COMs in the last years is related to the availability of greater and greater accurate radio telescopes. Previous theoretical or experimental spectroscopic characterization of these species is crucial for their eventual identification in space.

Among the COMs detected, it is found molecular species (isomers) that correspond to the same molecular formula, but contain different functional groups. In this way, examples of isomeric families characterized in space are acetic acid $\left(\mathrm{CH}_{3} \mathrm{COOH}\right.$; Mehringer et al. 1997), glycol aldehyde $\left(\mathrm{OHCH}_{2} \mathrm{CHO}\right.$; Hollis, Lovas \& Jewell 2000), and methyl formate $\left(\mathrm{CH}_{3} \mathrm{OCHO}\right.$; Brown et al. 1975; Churchwell \& Winnewisser 1975), or acetaldehyde $\left(\mathrm{CH}_{3} \mathrm{CHO}\right.$; Gottlieb 1973), ethylene oxide $\left(\mathrm{c}-\mathrm{C}_{2} \mathrm{H}_{4} \mathrm{O}\right.$; Dickens et al.
1997), and vinyl alcohol ( $\mathrm{CH}_{2} \mathrm{CHOH}$; Turner \& Apponi 2001), with molecular composition $[2 \mathrm{C}, 4 \mathrm{H}, 2 \mathrm{O}]$ and $[2 \mathrm{C}, 4 \mathrm{H}, \mathrm{O}]$, respectively. The knowledge of abundances and special distribution in the ISM of isomers could be useful to understand the formation routes, gas phase or grain process, of these molecules. In general, it is found that the most stable isomer should be the most abundant one (Lattelais et al. 2009). Nevertheless, since different chemical groups constitute the isomers, their formation routes could be different and, in these cases, the kinetic factors will be key on the abundances. From a kinetic point of view, activation barriers are determinant in the formation rate of the final product.

Acetamide and $\mathrm{N}$-metylformamide, two isomers with a peptidetype bond (-NH-CO-); have been detected in space (Hollis et al. 2006; Belloche et al. 2017). These molecules are very interesting from a prebiotic point of view, since the knowledge of their formation processes is essential to understand more complex molecules evolution. From the analysis of the relative abundances of both isomers, Belloche et al. (2017) suggest that the formation of these two molecules is controlled by kinetics rather than thermal equilibrium. They also remark that the detection of other isomers of molecular composition [2C, $5 \mathrm{H}, \mathrm{N}, \mathrm{O}]$ could clarify the origin of these systems.

On the other hand, considering the similarity found by Halfen, Ilyushin \& Ziurys (2011) between the abundances and distribution 

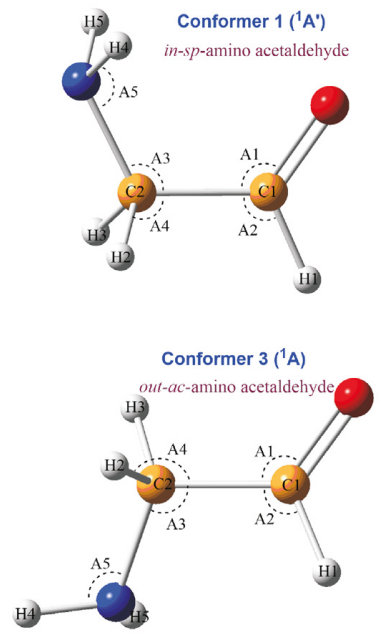

Figure 1. Structure of the four conformers located for amino acetaldehyde on the singlet potential energy surface (PES).

of formamide and acetamide towards $\mathrm{SgrB} 2(\mathrm{~N})$, they propose that acetamide could be synthetized through the reaction between formamide and $\mathrm{CH}_{5}^{+}$. In a recent study, we performed a theoretical study of this process (Redondo, Barrientos \& Largo 2014) and we found that the formation of protonated acetamide and amino acetaldehyde is an exothermic processe and takes place through barrier free paths. Therefore, this reaction could be a source of acetamide and amino acetaldehyde, a precursor of glycine by oxidation, in space. Amino acetaldehyde, an isomer of acetamide and $\mathrm{N}$-methylformamide, is located $\approx 26 \mathrm{kcal} \mathrm{mol}^{-1}$ above acetamide, the lowest energy structure obeying to the chemical formula [2C, $5 \mathrm{H}, \mathrm{N}, \mathrm{O}$ ] (Lattelais et al. 2010). In this theoretical study, amino acetaldehyde has been characterized at the density functional theory (DFT), in particular using the B3LYP functional. To the best of our knowledge, there have been no previous experimental studies on this species.

In order to help in the laboratory or astronomical detection of amino acetaldehyde, the main aim of this study is to characterize their conformers at the so-called 'spectroscopic' accuracy. To obtain this accuracy we employ a composite approach CCSD(T)/CBS + CV (Heckert, Kállay \& Gauss 2005; Heckert et al. 2006) that considers the extrapolation to the complete basis set limit, $\mathrm{CBS}$, and core-valence corrections, $\mathrm{CV}$, at the coupled clusters level including single and double excitations and a perturbative treatment of triple excitations, CCSD(T), level (Raghavachari et al. 1989). This approach obtain bond distances with an accuracy of $0.001-$ $0.002 \AA$ and angles accurate to 0.05-0.1 (Barone et al. 2013a).

\section{COMPUTATIONAL METHODS}

Different theoretical methodologies have been used within the context of DFT and ab initio approaches. Minima and transition states were characterized at the DFT level using the B3LYP (Becke 1986, 1988a,b; Lee, Yang \& Parr 1988) and the B2PLYP functionals (Grimme 2006). The second-order Møller-Plesset (MP2; Møller \& Plesset 1934) and coupled cluster with single and double excitations (CCSD; Raghavachari et al. 1989) ab initio theories were also used. In the CCSD calculations, the frozen-core (fc) approximation has been employed in conjunction with the Dunning's cc-pVTZ (Dunning 1989) basis set. At the B3LYP, B2PLYP, and MP2 levels of theory, the aug-cc-pVTZ (Dunning 1989; Kendall, Dunning \& Harrison 1992) basis set was used.

For each optimized structure, harmonic vibrational frequencies were calculated to characterize the stationary points and estimate the zero-point vibrational (ZPV) energies. In addition, anharmonic corrections were calculated at the CCSD/cc-pVTZ level and B2PLYP/aug-cc-pVTZ levels using a second-order perturbation treatment (VPT2; Barone 2005).

As in our previous work (Redondo, Largo \& Barrientos 2018), to obtain more accurate geometrical parameters, equilibrium rotational constants, and electronic energies for the amino acetaldehyde conformers, a composite procedure (Heckert et al. 2005, 2006) was applied. The computed predicted geometrical parameters and equilibrium rotational constants, which we will denote as $P$ (comp), are obtained using the following expression (Barone et al. 2013b):

$P($ comp $)=P(\mathrm{CBS})+\Delta P(\mathrm{CV})+\Delta P(\mathrm{~T})+\Delta P($ aug $)$,

with

$\Delta P(\mathrm{CV})=P(\mathrm{CCSD} / \mathrm{cc}-\mathrm{pCVTZ}$, all $)-P(\mathrm{CCSD} / \mathrm{cc}-\mathrm{pCVTZ}, \mathrm{fc})$,

$\Delta P(\mathrm{~T})=P(\mathrm{CCSD}(\mathrm{T}) / \mathrm{cc}-\mathrm{pVTZ})-P(\mathrm{CCSD} / \mathrm{cc}-\mathrm{pVTZ})$,

$\Delta P($ aug $)=P(C C S D /$ aug-cc-pVTZ $)-P(C C S D / c c-p V T Z)$,

where $P(\mathrm{CBS})$ considers the basis set truncation effects calculated employing the CCSD/cc-pVTZ and CCSD/cc-pVQZ data (Helgaker et al. 1997), $\Delta P(\mathrm{CV})$ is included to take into account corevalence electron correlation, and the terms $\Delta P(\mathrm{~T})$ and $\Delta P(\mathrm{aug})$ accounts for the correlation due to the inclusion of triplet excitation at the CCSD level and inclusion of diffuse functions (aug) in the basis set, respectively. Composite energies are estimated using the same approach including the term corresponding to ZPV energy.

Both GAUSSIAN 16 (Frisch at al. 2016) and CFOUR(Stanton et al. 2013) program packages were used for all computations.

\section{RESULTS AND DISCUSSION}

In this section, we will begin by the localization of the amino acetaldehyde conformers and the analysis of their stability. Then we will discuss the interconversion processes between them and finally we will report their spectroscopic properties.

\subsection{Amino acetaldehyde conformers}

In Fig. 1, we show the structure of the four conformers characterized for amino acetaldehyde on the singlet potential energy surface (PES). Conformer 1 has $\mathrm{C}_{\mathrm{s}}$ symmetry with ${ }^{1} \mathrm{~A}$ ' electronic ground state, and the other three conformers have no symmetry and their ground state is ${ }^{1} \mathrm{~A}$. As can be seen in Fig. 1, conformers 1 and 2 , which have the oxygen atom and the $\mathrm{NH}_{2}$ group on the same side of the plane with a $\mathrm{OC} 1 \mathrm{C} 2 \mathrm{~N}$ dihedral angle near $0^{\circ}$, reveal a synperiplanar $(s p)$ arrangement. In conformer 1 the $\mathrm{H}$ atoms of the $\mathrm{NH}_{2}$ group are pointing in the direction of the oxygen (in), whereas in conformer 2 the hydrogen atoms are addressed outside the skeleton of the molecule (out) and we can denote them as in$s p$ - and $o u t-s p$-amino acetaldehyde, respectively. Conformers 3 and 4 , which arise from conformer 1 or 2 by rotation around the $\mathrm{C}$ $\mathrm{C}$ bond, with a OC1C2N dihedral angle between $90^{\circ}$ and $150^{\circ}$ or $-90^{\circ}$ and $-150^{\circ}$, are called anticlinal $(a c)$. Considering the orientation of hydrogen in the $\mathrm{NH}_{2}$ group, conformer 3 is denoted as 
Table 1. Structural parameters (distances in Angstroms and angles in degrees) for the amino acetaldehyde conformers at different levels of theory.

\begin{tabular}{|c|c|c|c|c|c|c|c|c|c|c|c|c|c|c|c|}
\hline Level & $\mathrm{OC} 1$ & $\mathrm{C} 1 \mathrm{C} 2$ & $\mathrm{~A} 1$ & $\mathrm{C} 1 \mathrm{H} 1$ & A2 & $\mathrm{C} 2 \mathrm{~N}$ & A3 & $\mathrm{H} 2 \mathrm{C}$ & A4 & $\mathrm{H} 4 \mathrm{~N}$ & A5 & $\operatorname{Dih} 1^{a}$ & $\operatorname{Dih} 2^{b}$ & $\operatorname{Dih} 3^{c}$ & $\operatorname{Dih} 4^{d}$ \\
\hline B3LYP/aug-cc-pVTZ & 1.2040 & 1.5150 & 124.7 & 1.1113 & 114.6 & 1.4474 & 117.4 & 1.0964 & 107.0 & 1.0129 & 110.4 & 180.0 & 0.0 & -124.1 & -58.5 \\
\hline B2PLYP/aug-cc-pVTZ & 1.2086 & 1.5129 & 124.4 & 1.1080 & 114.9 & 1.4474 & 117.0 & 1.0944 & 107.1 & 1.0123 & 110.0 & 180.0 & 0.0 & -123.9 & -58.0 \\
\hline $\mathrm{CCSD} / \mathrm{cc}-\mathrm{pVTZ}$ & 1.2052 & 1.5137 & 124.0 & 1.1059 & 115.9 & 1.4498 & 116.2 & 1.0940 & 107.4 & 1.0123 & 108.9 & 180.0 & 0.0 & -123.6 & -56.8 \\
\hline Composite & 1.2050 & 1.5104 & 124.1 & 1.1061 & 113.3 & 1.4430 & 116.5 & 1.0940 & 107.1 & 1.0111 & 110.1 & 180.0 & 0.0 & -123.7 & -58.1 \\
\hline MP2/aug-cc-pVTZ & 1.2151 & 1.5018 & 123.7 & 1.1035 & 115.4 & 1.4478 & 111.6 & 1.0993 & 105.3 & 1.0140 & 109.2 & 179.0 & 0.0 & -115.2 & -24.5 \\
\hline B2PLYP/aug-cc-pVTZ & 1.2094 & 1.5029 & 124.0 & 1.1045 & 115.0 & 1.4476 & 112.2 & 1.0997 & 105.3 & 1.0125 & 109.8 & 179.2 & 9.3 & -116.0 & -22.4 \\
\hline $\mathrm{CCSD} / \mathrm{cc}-\mathrm{pVTZ}$ & 1.2053 & 1.5066 & 123.9 & 1.1031 & 115.2 & 1.4506 & 111.5 & 1.0994 & 105.5 & 1.0115 & 108.6 & 180.0 & 10.8 & -114.6 & -28.1 \\
\hline Composite & 1.2057 & 1.5010 & 123.7 & 1.1030 & 115.4 & 1.4445 & 112.0 & 1.0983 & 105.7 & 1.0118 & 109.9 & 180.0 & 8.2 & -116.8 & -19.0 \\
\hline \multicolumn{16}{|l|}{ Conformer 3} \\
\hline B3LYP/aug-cc-pVTZ & 1.2037 & 1.5159 & 124.2 & 1.1086 & 114.2 & 1.4583 & 110.9 & 1.0951 & 105.7 & 1.0100 & 111.8 & 179.4 & 149.8 & -93.0 & 159.3 \\
\hline \multicolumn{16}{|l|}{ Conformer 4} \\
\hline B3LYP/aug-cc-pVTZ & 1.2036 & 1.5175 & 125.0 & 1.1134 & 114.4 & 1.4589 & 114.3 & 1.0922 & 108.3 & 1.0121 & 112.0 & 178.4 & -134.8 & 122.0 & 60.8 \\
\hline MP2/aug-cc-pVTZ & 1.2142 & 1.5109 & 124.8 & 1.1089 & 114.6 & 1.4586 & 113.8 & 1.0906 & 108.3 & 1.0126 & 111.1 & 178.5 & -134.1 & 121.7 & 59.7 \\
\hline B2PLYP/aug-cc-pVTZ & 1.2082 & 1.5147 & 124.9 & 1.1103 & 114.5 & 1.4588 & 114.0 & 1.0905 & 108.3 & 1.0115 & 111.6 & 178.4 & -134.3 & 121.9 & 60.4 \\
\hline CCSD/cc-pVTZ & 1.2042 & 1.5162 & 124.6 & 1.1086 & 114.6 & 1.4579 & 114.7 & 1.0908 & 108.0 & 1.0112 & 110.6 & 180.0 & -142.1 & 123.3 & 57.2 \\
\hline Composite & 1.2047 & 1.5122 & 124.4 & 1.1088 & 115.1 & 1.4532 & 114.3 & 1.0905 & 108.1 & 1.0100 & 111.9 & 180.0 & -138.1 & 123.0 & 58.3 \\
\hline
\end{tabular}

${ }^{a} \operatorname{Dih} 1=\operatorname{dih}(\mathrm{C} 2 \mathrm{C} 1 \mathrm{OH} 1)$.

${ }^{b} \operatorname{Dih} 2=\operatorname{dih}(\mathrm{NC} 2 \mathrm{C} 3 \mathrm{O})$.

${ }^{c} \operatorname{Dih} 3=\operatorname{dih}(\mathrm{H} 2 \mathrm{C} 2 \mathrm{C} 1 \mathrm{O})$.

${ }^{d} \operatorname{Dih} 4=\operatorname{dih}(\mathrm{H} 4 \mathrm{NC} 2 \mathrm{C} 1)$

Table 2. Relative energies, in $\mathrm{kcal} \mathrm{mol}^{-1}$, for the four conformers of amino acetaldehyde and transition states connecting them computed at different levels of theory (ZPV energies included).

\begin{tabular}{lcccccccc}
\hline Level & Conf. 1 & Conf. 2 & Conf. 3 & Conf. 4 & TS21 & TS41 & TS32 & TS43 \\
\hline B3LYP/aug-cc-pVTZ & 0.00 & 0.56 & 1.53 & 1.78 & 0.48 & 3.36 & 4.04 & 3.57 \\
MP2/aug-cc-pVTZ & 0.00 & 0.68 & 1.67 & 2.01 & 0.62 & 3.39 & 3.68 & 4.43 \\
B2PLYP/aug-cc-pVTZ & 0.00 & 0.63 & 1.57 & 1.86 & 0.56 & 3.34 & 3.86 & 3.93 \\
CCSD/cc-pVTZ & 0.00 & 1.11 & 1.66 & 2.00 & 1.04 & 3.50 & 3.76 & 5.25 \\
CCSD(T)/cc-pVTZ & 0.00 & 1.07 & 1.78 & 1.98 & & & & \\
CCSD/cc-pVQZ & 0.00 & 0.91 & 1.56 & 1.95 & & & \\
CCSD/aug-cc-pVTZ & 0.00 & 0.86 & 1.49 & 1.90 & & & & \\
CCSD(T)/aug-cc-pVQZ// & & & & & & & & \\
CCSD/cc-pVTZ & 0.00 & 0.78 & 1.63 & 1.92 & 0.68 & 3.29 & 3.62 & 4.54 \\
Composite & 0.00 & 0.44 & 1.44 & 1.81 & & & & \\
\hline
\end{tabular}

out-ac-amino acetaldehyde, and conformer 4 denoted as in-acamino acetaldehyde.

The geometrical parameters of the low-lying conformers of amino acetaldehyde computed at different levels of theory are shown in Table 1, whereas in Table 2 their relative energies calculated at the same levels of theory are included. Since the structure of the four conformers only differs in the relative orientation of the functional groups, the bond lengths are very close in the four minima. The most significant change is found for the $\mathrm{C}-\mathrm{N}$ bond length that is about $0.01 \AA$ shorter in synperiplanar conformers (1 and 2) than in anticlinal ones (3 and 4). If we compare the different levels of calculations employed with the composite values, we can see in Table 1 that the differences found in the bond lengths are in the hundredths of angstroms, and for the angles are less than $1^{\circ}$. The greatest discrepancies correspond to the dihedral angles, specifically in the case of the Dih2 in conformer 2.
Regarding the relative energies of amino acetaldehyde conformers shown in Table 2, all levels of theory used in this work provide the same stability order of conformers. The deviation obtained for the relative energies calculated at different levels of theory is for the four conformers less than $0.7 \mathrm{kcal} \mathrm{mol}^{-1}$. The values obtained at the CCSD(T)/aug-cc-pVQZ level on the CCSD/cc-pVTZ geometries (denoted as CCSD(T)/aug-cc-pVQZ//CCSD/cc-pVTZ) are much closer to those estimated with the composite approach than the CCSD/cc-pVTZ ones. It should be pointed out that relative values obtained at the B3LYP and especially at the B2PLYP levels for this system are very close to the composite ones. Therefore, these levels could be employed for predicting relative energies in larger systems.

All levels of theory agree in the prediction of conformer 1 , in-spamino acetaldehyde, as the most stable one. The other synperiplanar conformer, out-sp-amino acetaldehyde, the following in energy, is 


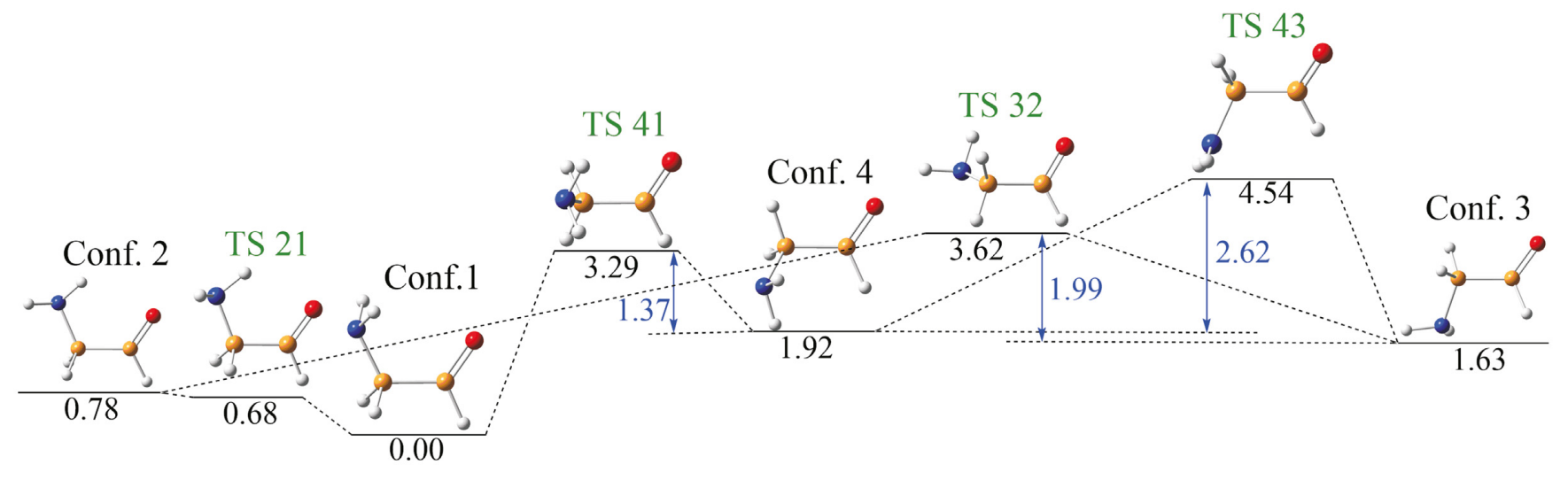

Figure 2. Interconversion processes between the four conformers located for amino acetaldehyde. Relative energies (in black) respect to conformer 1, and interconversion barriers (in blue), calculated at the CCSD(T)/aug-cc-pVQZ//CCSD/cc-pVTZ level. Energies in kcal mol ${ }^{-1}$ (ZPV energies are included).

located only $0.44 \mathrm{kcal} \mathrm{mol}^{-1}$ above conformer 1 at the composite level. Anticlinal conformation is less stable than synperiplanar one, so conformers 3 and 4 are the most energetics. The energy difference between the two anticlinal conformers is only $0.37 \mathrm{kcal} \mathrm{mol}^{-1}$ at the composite level, similar to that found for synperiplanar ones. The stability of synperiplanar conformers (1 and 2) over anticlinal ones ( 3 and 4) is related to the interaction between the hydrogen atoms of the group $\mathrm{NH}_{2}$ and the oxygen one that is greater in conformer 1 . However, for anticlinal structures steric factors make conformer 3 slightly more stable than conformer 4 , due to the distinct orientation of the hydrogen atoms of the $\mathrm{NH}_{2}$ group with respect to the heavy atoms skeleton.

\subsection{Isomerization processes}

Once we have characterized the different conformers of amino acetaldehyde we will now analyse the interconversion processes between them and discuss the results in the context of the ISM. For that, we have located the transition states associated with the interconversion processes among the four conformers. The relative energies of transition states for the different isomerization processes between conformers are included also in Table 2, and in Fig. 2, we represent the different isomerization processes including the isomerization energies. These energies are calculated at the $\operatorname{CCSD}(\mathrm{T}) /$ augcc-pVQZ on the optimized CCSD/cc-pVTZ geometries. This level is shown in previous section that provides relative energies close to that calculated using the composite approach. We denote transition states with TS followed by two numbers that correspond to the conformers they connect. Transition states structures are supported by vibrational analysis, each one presents one imaginary frequency associated with the conversion process. With regard to the geometrical parameters on the optimized transition state structures, as can be observed in Fig. 2, all of them are intermediate between those of the conformers that connect the transition state structures.

We can see in Fig. 2 that the isomerization of the conformer 2, out-sp-amino acetaldehyde, to the most stable conformer 1 , in$s p$-amino acetaldehyde, takes place through transition state TS21, where a rotation of the $\mathrm{NH}_{2}$ group occurs, and does not imply a net activation barrier. Transition state TS21 is located below the conformer $2\left(0.10 \mathrm{kcal} \mathrm{mol}^{-1}\right.$ at the $\operatorname{CCSD}(\mathrm{T}) /$ aug-cc-pVQZ level). The same result is obtained for all the calculation levels used, as can be seen in Table 2. Therefore, conformer 2 evolves directly to the conformer 1 and its experimental observation would not be possible. The isomerization between the two anticlinal conformers (3 and 4) takes place through transition state TS43 that is located $2.62 \mathrm{kcal} \mathrm{mol}^{-1}(\approx 1320 \mathrm{~K})$ above the conformer 4 , in-ac-amino acetaldehyde. Isomer 4 can also isomerize to the more stable isomer 1 through the transition state TS41. In this process a rotation around the $\mathrm{C}-\mathrm{C}$ bond occurs. The interconversion barrier associated with the conformer $4 \longrightarrow$ conformer 1 process is about $1.37 \mathrm{kcal} \mathrm{mol}^{-1}(\approx 690 \mathrm{~K})$ above conformer 4 . Therefore, the most favourable isomerization process for conformer 4 , in-ac-amino acetaldehyde, from thermodynamic and kinetic points of view, is the interconversion into the most stable conformer 1 , in-sp-amino acetaldehyde. Finally, conformer 3, out-ac-amino acetaldehyde, can isomerize to conformer 2, out-sp-amino acetaldehyde. This process presents an activation barrier associated with transition state TS32 about $1.99 \mathrm{kcal} \mathrm{mol}^{-1}(\approx 1000 \mathrm{~K})$. Once conformer 2 is reached, it evolves to the most stable conformer, in-sp-amino acetaldehyde. Thus, the isomerization of the conformer 3 to 1 is not a direct process since it takes place through the conformer 2 .

The obtained energy barriers for the interconversion processes among the four conformers show that, at the low temperatures of the ISM, conformer 2 will isomerize to conformer 1 . The isomerization processes between the conformers 1, 3, and 4 involve net energy barriers. The easiest process corresponds to the interconversion of conformer 4 (the less stable) to conformer 1 (the most stable) with a barrier of $1.37 \mathrm{kcal} \mathrm{mol}^{-1}(\approx 690 \mathrm{~K})$. Therefore, it is worth noticing that the three conformers, 1,3 , and 4 , could coexist in the ISM.

\subsection{Spectroscopic parameters}

Besides the conformational landscape of amino acetaldehyde, we report high-level calculated spectroscopic parameters relevant to the investigation of the experimental infrared (IR) and rotational spectrum, which are collected in Tables 3 and 4. The composite approach was used to compute the equilibrium rotational constants $\left(\mathrm{A}_{\mathrm{e}}, \mathrm{B}_{\mathrm{e}}, \mathrm{C}_{\mathrm{e}}\right.$ ), while the corresponding constants for the ground vibrational state $\left(\mathrm{A}_{0}, \mathrm{~B}_{0}, \mathrm{C}_{0}\right)$ were obtained from the vibrationrotation coupling constants and degeneracy factors for the vibrational modes. The similarities found in structural parameters, given in Table 1, between the two conformations with a synperiplanar disposition (conformers 1 and 2) or with anticlinal orientation (conformers 3 and 4) remain in the calculated rotational constants (Table 3 ). We have also presented in Table 3 centrifugal distortion parameters in the symmetrically reduced Hamiltonian $\left(\Delta_{J}, \Delta_{K}\right.$, $\Delta_{J K}, \delta_{J}$, and $\delta_{k}$ ). In this case, another interesting spectroscopic tool is taken into account: the presence of a ${ }^{14} \mathrm{~N}$ nucleus. Therefore, 
Table 3. Spectroscopic parameters for the amino acetaldehyde conformers (in $\mathrm{MHz}$ ). Equilibrium rotational constants $\left(\mathrm{A}_{\mathrm{e}}, \mathrm{B}_{\mathrm{e}}, \mathrm{C}_{\mathrm{e}}\right)$ and rotational constants for the ground vibrational state $\left(\mathrm{A}_{0}, \mathrm{~B}_{0}, \mathrm{C}_{0}\right)$ are computed with the composite method. Centrifugal distortion parameters in the symmetrically reduced Hamiltonian $\left(\Delta_{J}, \Delta_{K}, \Delta_{J K}, \delta_{J}\right.$, and $\left.\delta_{k}\right)$, the diagonal elements of the ${ }^{14} \mathrm{~N}$ nuclear quadrupole coupling tensor $\left(\chi_{a a}, \chi_{b b}, \chi_{c c}\right)$, and dipole moment components ( $\mu$ in Debye) are computed at the CCSD/cc-pVTZ level.

\begin{tabular}{lcccc}
\hline & Confor. 1 & Confor. 2 & Confor. 3 & Confor. 4 \\
\hline $\mathrm{A}_{\mathrm{e}}$ & 17849.4 & 17962.9 & 32345.9 & 30429.1 \\
$\mathrm{~B}_{\mathrm{e}}$ & 6029.6 & 6290.3 & 4348.3 & 4349.4 \\
$\mathrm{C}_{\mathrm{e}}$ & 4747.3 & 4832.0 & 4131.7 & 4158.1 \\
$\mathrm{~A}_{0}$ & 17762.9 & 17869.9 & 33197.7 & 34367.9 \\
$\mathrm{~B}_{0}$ & 5973.8 & 6199.7 & 4294.6 & 4185.2 \\
$\mathrm{C}_{0}$ & 4704.5 & 4772.2 & 4057.6 & 3929.7 \\
$\Delta_{J} \times 10^{3}$ & 5.64515 & 5.07310 & 2.94212 & 12.5669 \\
$\Delta_{J K} \times 10^{3}$ & -15.8292 & -18.0168 & -100.718 & -476.493 \\
$\Delta_{K} \times 10^{3}$ & 47.9392 & 51.5130 & 1993.72 & 6520.91 \\
$\delta_{J} \times 10^{3}$ & -1.62698 & -1.54878 & 0.33183 & 1.85029 \\
$\delta_{K} \times 10^{6}$ & -28.4672 & -107.309 & -42.0461 & -49.5764 \\
$\mu_{a}$ & 0.26 & 1.23 & 2.83 & 1.67 \\
$\mu_{b}$ & 1.64 & 2.18 & 0.63 & 1.19 \\
$\mu_{c}$ & 0.0 & 1.24 & 0.11 & 0.8 \\
$\chi_{a a}$ & -4.3467 & -0.5322 & 1.2532 & 0.8991 \\
$\chi_{b b}-\chi_{c c}$ & 0.5809 & -4.5809 & -2.2590 & 4.5104 \\
\hline
\end{tabular}

we give the values of the quadrupole coupling constants for the ${ }^{14} \mathrm{~N}$ nucleus $\left(\chi_{a a}, \chi_{b b}\right.$, and $\left.\chi_{c c}\right)$. On the one hand, the rotational constants provide information of the mass distribution. In addition, the nuclear quadrupole coupling interactions show a strong dependence on the electronic environment close of the ${ }^{14} \mathrm{~N}$ nucleus. It introduces hyperfine rotational signatures at located sites of amino acetaldehyde and acts as a conclusive probe of the position, orientation, and chemical environment of the ${ }^{14} \mathrm{~N}$ quadrupolar nucleus. Hence, to identify the plausible observed species, the resolution of the nuclear quadrupole hyperfine structure of amino acetaldehyde is needed. Moreover, the values of the computed dipole moment components (Table 3) are high enough to allow for the observation of these conformers by rotational spectroscopy.

To guide spectral searches, we have simulated the rotational spectrum from the corresponding rotational constants, dipole-moment components, and ${ }^{14} \mathrm{~N}$ nuclear quadrupole coupling tensor constants. In Fig. 3, we present a prediction of the rotational spectra of in-spamino acetaldehyde (conformer 1) in the $0-500 \mathrm{GHz}$ frequency region using a semirigid-rotor approximation including quartic centrifugal distortion parameters in the symmetrically reduced Hamiltonian. SPCAT program (Pickett 1991) is employed to predict frequencies and intensities from the computed parameters.

In Table 4, we give CCSD harmonic and anharmonic vibrational frequencies and the corresponding IR intensities for the four amino acetaldehyde conformers. These results could guide their eventual characterization in the gas phase through IR spectroscopy. We were only able to evaluate anharmonic corrections at the CCSD/ccpVTZ level have only been possible to be evaluated for in-sp-amino acetaldehyde (conformer 1) that has $\mathrm{C}_{\mathrm{s}}$ symmetry. For this isomer, we have also computed the anharmonic corrections at the B2PLYP and MP2/aug-cc-pVTZ levels in order to check the accuracy of both approaches. Our results show that anharmonic corrections at the B2PLYP/aug-cc-pVTZ are closer to those calculated at the CCSD level than when the corrections are calculated at the MP2 level. The mean absolute errors with respect to CCSD frequencies are 40 and $14 \mathrm{~cm}^{-1}$ for MP2 and B2PLYP anharmonic corrections, respectively. Therefore, for the rest of conformers, with $\mathrm{C}_{1}$ symmetry, anharmonic corrections are estimated at the B2PLYP/aug-cc-pVTZ level.

Anharmonic frequencies, in the most of the cases, are lower than the corresponding harmonic ones, according with the usual behaviour. The largest anharmonic corrections are found, in general, in the larger frequencies corresponding to stretching modes. As we can see in Table 4, there is not an evident relation between anharmonic and harmonic vibrational frequencies. Hence, it is not

Table 4. Harmonic, $\omega$, and anharmonic, $v$, vibrational frequencies $\left(\mathrm{cm}^{-1}\right)$, and IR intensities $\left(\mathrm{km} \mathrm{mol}^{-1}\right)$ for the amino acetaldehyde conformers calculated at the CCSD/cc-pVTZ level.

\begin{tabular}{|c|c|c|c|c|c|c|c|c|c|c|c|c|c|c|c|c|}
\hline \multirow[b]{2}{*}{ Mode } & \multicolumn{4}{|c|}{ Conformer 1} & \multicolumn{4}{|c|}{ Conformer $2^{a}$} & \multicolumn{4}{|c|}{ Conformer $3^{a}$} & \multicolumn{4}{|c|}{ Conformer $4^{a}$} \\
\hline & $\omega$ & $I_{\text {har }}$ & $v$ & $I_{\text {anhar }}$ & $\omega$ & $I_{\text {har }}$ & $v$ & $I_{\text {anhar }}$ & $\omega$ & $I_{\text {har }}$ & $v$ & $I_{\text {anhar }}$ & $\omega$ & $I_{\text {har }}$ & $v$ & $I_{\text {anhar }}$ \\
\hline$a^{\prime}, a$ & 125 & 47.2 & 144 & 79.9 & 143 & 39.9 & 29 & 13.7 & 84 & 10.2 & 85 & 9.2 & 58 & 9.6 & 28 & 6.6 \\
\hline$a^{\prime}, a$ & 268 & 21.7 & 269 & 19.5 & 295 & 7.1 & 461 & 9287.2 & 352 & 14.1 & 348 & 10.3 & 346 & 9.3 & 341 & 21.8 \\
\hline$a^{\prime}, a$ & 708 & 0.5 & 714 & 0.4 & 701 & 24.3 & 677 & 23.1 & 537 & 5.3 & 536 & 4.5 & 516 & 9.3 & 527 & 8.3 \\
\hline$a^{\prime}, a$ & 740 & 2.7 & 725 & 2.2 & 763 & 5.2 & 754 & 59.8 & 762 & 8.9 & 730 & 14.6 & 750 & 0.5 & 652 & 1.2 \\
\hline$a^{\prime}, a$ & 868 & 64.5 & 845 & 86.3 & 857 & 105.0 & 792 & 59.7 & 873 & 136.6 & 807 & 91.6 & 864 & 192.6 & 769 & 157.0 \\
\hline$a^{\prime}, a$ & 1174 & 13.5 & 1143 & 8.8 & 1148 & 3.0 & 1126 & 4.8 & 1131 & 4.8 & 1095 & 6.5 & 1137 & 5.1 & 1121 & 12.9 \\
\hline$a^{\prime}, a$ & 1222 & 0.1 & 1202 & 0.5 & 1245 & 9.0 & 1205 & 7.1 & 1210 & 0.9 & 1186 & 0.3 & 1215 & 3.5 & 1189 & 0.0 \\
\hline$a^{\prime}, a$ & 1393 & 4.5 & 1356 & 4.3 & 1275 & 0.9 & 1251 & 1.9 & 1327 & 13.1 & 1289 & 14.1 & 1357 & 8.5 & 1321 & 13.1 \\
\hline$a^{\prime}, a$ & 1407 & 0.6 & 1370 & 0.9 & 1434 & 10.2 & 1397 & 14.0 & 1398 & 0.7 & 1366 & 1.4 & 1396 & 0.7 & 1365 & 0.1 \\
\hline$a^{\prime}, a$ & 1429 & 11.3 & 1405 & 4.2 & 1449 & 4.8 & 1410 & 3.3 & 1438 & 8.3 & 1403 & 3.7 & 1436 & 3.3 & 1397 & 3.2 \\
\hline$a^{\prime}, a$ & 1476 & 10.6 & 1438 & 7.4 & 1510 & 10.8 & 1474 & 0.0 & 1500 & 5.5 & 1456 & 4.6 & 1485 & 4.3 & 1433 & 2.4 \\
\hline$a^{\prime}, a$ & 3086 & 13.0 & 2935 & 14.0 & 3059 & 29.7 & 2885 & 32.5 & 3093 & 18.7 & 2950 & 15.8 & 3125 & 10.8 & 2974 & 15.8 \\
\hline$a^{\prime}, a$ & 3532 & 1.5 & 3383 & 0.5 & 3549 & 4.9 & 3388 & 5.6 & 3547 & 0.7 & 3400 & 0.3 & 3535 & 0.8 & 3392 & 0.0 \\
\hline$a^{\prime}, a$ & 3603 & 3.0 & 3431 & 1.4 & 3642 & 9.9 & 3467 & 4.7 & 3630 & 3.8 & 3464 & 0.5 & 3620 & 2.1 & 3452 & 0.0 \\
\hline
\end{tabular}

${ }^{a}$ Anharmonic contributions are computed at the B2PLYP/aug-cc-pVTZ level. 


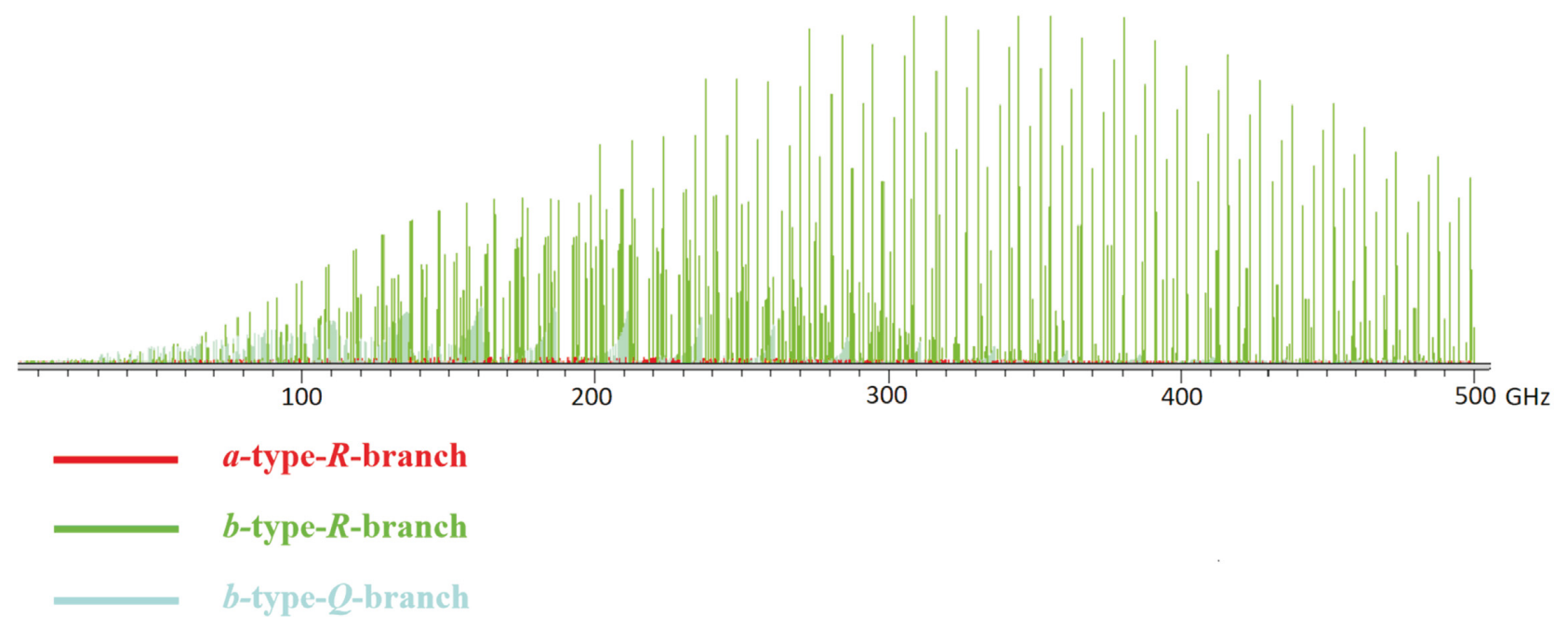

Figure 3. Calculated rotational spectrum at $50 \mathrm{~K}$ of in-sp-amino acetaldehyde (conformer 1 ) in the $0-500 \mathrm{GHz}$ frequency region using a semirigid-rotor approximation including quartic centrifugal distortion parameters in the symmetrically reduced Hamiltonian.

possible to found a general scale factor to bring the harmonic frequencies to the anharmonic ones. The same situation is found when we compare anharmonic intensities with the harmonic ones. Thus, we can infer that in order to predict reliable IR spectra for these conformers it is mandatory to include anharmonic contributions.

The band associated with the $\mathrm{C}-\mathrm{O}$ stretching mode is one of the most intense in the IR spectrum of all conformers. This band is located between $1806 \mathrm{~cm}^{-1}$ in conformer 3 and $1869 \mathrm{~cm}^{-1}$ in conformer 1 . The two anticlinal conformers, 3 and 4, present an IR spectrum similar. It is related with the very close geometries, which only differ in the orientation of the hydrogen atoms of the $\mathrm{NH}_{2}$ group.

\section{CONCLUSIONS}

In this work, we have performed a study of amino acetaldehyde conformers using different quantum chemical approaches. We have characterized four low-lying conformers on the singlet PES. Conformers 1 and 2 have a synperiplanar $(s p)$ arrangement with the carboxylic oxygen atom and the $\mathrm{NH}_{2}$ group on the same side of the plane. In conformer 1 , denoted as in-sp-amino acetaldehyde, the $\mathrm{H}$ atoms of the $\mathrm{NH}_{2}$ group point in the direction of the oxygen, whereas in conformer 2 the hydrogen atoms are addressed outside the skeleton of the molecule and is called out-sp-amino acetaldehyde. Conformers 3 and 4 present an anticlinal $(a c)$ disposition of the carboxylic oxygen atom and the $\mathrm{NH}_{2}$ group. Considering the orientation of hydrogen in the $\mathrm{NH}_{2}$ group, conformer 3 is denoted as out-ac-amino acetaldehyde, and conformer 4 as in- $a c$-amino acetaldehyde.

The same stability order of conformers is obtained at all levels of theory used in this work. The variations of the relative energies computed at the different levels are less than $0.7 \mathrm{kcal} \mathrm{mol}^{-1}$. Conformer 1, in-sp-amino acetaldehyde, is the most stable followed by the other synperiplanar conformer, out-sp-amino acetaldehyde, which is located only $0.44 \mathrm{kcal} \mathrm{mol}^{-1}$ above conformer 1 at the composite level. Synperiplanar conformers (1 and 2) are more stable than anticlinal ones ( 3 and 4 ) due to the interaction between the hydrogen atom of the group $\mathrm{NH}_{2}$ and the oxygen one. Conformers 3 and 4 are located 1.44 and $1.81 \mathrm{kcal} \mathrm{mol}^{-1}$ above the conformer 1 at the composite level, respectively.
Isomerization processes between the four conformers are also studied. The obtained energy barriers for the interconversion processes show that, at the low temperatures of the ISM, the isomerization of conformer 2 to conformer 1 (the most stable) is a barrierfree process, therefore, conformer 2 will isomerize to 1 and its experimental observation would not be possible. The isomerization processes between the conformers 1, 3, and 4 involve a net energy barrier. The easiest process corresponds to the interconversion of conformer 4 (the less stable) to conformer 1 (the most stable) with a barrier of $1.37 \mathrm{kcal} \mathrm{mol}^{-1}(\approx 690 \mathrm{~K})$ at the $\mathrm{CCSD}(\mathrm{T}) / \mathrm{aug}$-ccpVQZ//CCSD/cc-pVTZ level. However, under the conditions of the ISM the three conformers, 1, 3, and 4, could coexist.

For the four conformers we have obtained the relevant spectroscopic parameters to rotational spectroscopy with 'spectroscopic' accuracy at the composite level. We provided accurate theoretical values of the ${ }^{14} \mathrm{~N}$ nuclear quadrupole coupling constants together with the rotational constants in order to reproduce the spectrum to enable its distinct possible identification. For observations in low-frequency regions like the ones accessible for the Green Bank Telescope (GBT), the hyperfine components of the transitions, which normally are propagated several $\mathrm{MHz}$, could transpire to be a handicap for interpreting the radioastronomical observations. Vibrational frequencies and IR intensities are also reported at the CCSD level including anharmonic corrections.

These results could help in the experimental characterization of amino acetaldehyde in the laboratory or in space by radio or IR spectroscopy.

\section{ACKNOWLEDGEMENTS}

Financial support from the Spanish Ministerio de Economía Industria y Competitividad (Grant AYA2017-87515-P) and the Junta de Castilla y León (Grant VA010G18) is gratefully acknowledged. MS-N acknowledges funding from a Predoctoral FPU Grant (FPU17/02987).

\section{REFERENCES}

Barone V., 2005, J. Chem. Phys., 122, 014108

Barone V., Biczysko M., Bloino J., Puzzarini C., 2013a, Phys. Chem. Chem. Phys., 15, 1358 
Barone V., Biczysko M., Bloino J., Puzzarini C., 2013b, J. Chem. Theory Comput., 9, 1533

Becke A. D., 1986, J. Chem. Phys., 84, 4524

Becke A. D., 1988a, J. Chem. Phys., 88, 1053

Becke A. D., 1988b, J. Chem. Phys., 88, 2547

Belloche A. et al., 2017, A\&A, 601, A49

Brown R. D., Crofts J. G., Gardner F. F., Godfrey P. D., Robinson B. J., Whiteoak J. B., 1975, ApJ, 197, L29

Churchwell E., Winnewisser G., 1975, A\&A, 45, 229

Dickens J. E., Irvine W. M., Ohishi M., Ikeda M., Ishikawa S., Nummelin A., Hjalmarson ̊., 1997, ApJ, 489, 753

Dunning T. H., 1989, J. Chem. Phys., 90, 1007

Frisch M. J. et al., 2016, Gaussian 16. Gaussian, Inc., Wallingford, CT

Gottlieb C. A., 1973, in Gordon M. A., Snyder L. E., eds, Molecules in the Galactic Environment. Wiley, New York, p. 181

Grimme S., 2006, J. Chem. Phys., 124, 034108

Halfen D. T., Ilyushin V. V., Ziurys L. M., 2011, ApJ, 743, 60

Heckert M., Kállay M., Gauss J., 2005, Mol. Phys., 103, 2109

Heckert M., Kállay M., Tew D. P., Klopper W., Gauss J., 2006, J. Chem. Phys., 125, 044108

Helgaker T., Klopper W., Koch H., Noga J., 1997, J. Chem. Phys., 106, 9639

Hollis J. M., Lovas F. J., Jewell P. R., 2000, ApJ, 540, L107
Hollis J. M., Lovas F. J., Remijan A. J., Jewell P. R., Ilyushin V. V., Kleiner I., 2006, ApJ, 643, L25

Kendall R. A., Dunning T. H., Harrison R. J., 1992, J. Chem. Phys., 96, 6796

Lattelais M., Pauzat F., Ellinger Y., Ceccarelli C., 2009, ApJ, 696, L133

Lattelais M., Pauzat F., Ellinger Y., Ceccarelli C., 2010, A\&A, 519, A30

Lee C., Yang W., Parr R. G., 1988, Phys. Rev. B (Condensed Matter), 37, 785

Mehringer D. M., Snyder L. E., Miao Y., Lovas F., 1997, ApJ, 480, L71

Møller C., Plesset M., 1934, Phys. Rev., 46, 618

Pickett H. M., 1991, J. Mol. Spectrosc., 148, 371

Quan D., Herbst E., Corby J. F., Durr A., Hassel G., 2016, ApJ, 824, 129

Raghavachari K., Trucks G. W., Pople J. A., Head-Gordon M., 1989, Chem. Phys. Lett., 157, 479

Redondo P., Barrientos C., Largo A., 2014, ApJ, 793, 32

Redondo P., Largo A., Barrientos C., 2018, MNRAS, 478, 3042

Stanton J. F., Gauss J., Harding M. E., Szalay P. G., 2013, CFOUR, a quantum chemical program package

Turner B. E., Apponi A. J., 2001, ApJ, 561, L207

This paper has been typeset from a $\mathrm{T}_{\mathrm{E}} \mathrm{X} / \mathrm{LTT}_{\mathrm{E}} \mathrm{X}$ file prepared by the author. 